\title{
Entre acadianité et mondialisation : l'expression du « soi-Acadien » à la rencontre de l'autre dans Une lettre au bout du monde de Jean-Philippe Raîche
}

\author{
Jimmy Thibeault \\ Université Sainte-Anne
}

Pour les générations de poètes acadiens qui sont arrivées à l'écriture à la fin des années 1990 et au cours des années 2000, il semble difficile, voire impossible, de sortir de l'ombre des poètes qui, au cours de la période qu'Emir Delic et moi-même avons nommée la « longue décennie 1970 (DELIC et ThiBEAUlt, 2014-2015), ont fait entrer, avec un retentissant « cri

\footnotetext{
${ }^{1}$ La longue décennie 1970 couvre la période allant de 1968 à 1985 et correspond à une période de prise de parole des différentes communautés francophones du Canada dont l'objectif était d'affirmer leurs particularités et leur inscription dans la modernité.
} 
JIMMY THIBEAULT, «Entre acadianité et mondialisation : 1'expression du " soiAcadien " à la rencontre de l'autre dans Une lettre au bout du monde de Jean-Philippe Raîche »

de terre », la poésie acadienne dans sa modernité. Encore aujourd'hui, en effet, résonnent haut et fort la voix des poètes phares qui ont rompu en leur temps avec une Acadie traditionnelle, qu'ils considéraient comme folklorique et passéiste, pour faire entendre une parole qui se réclamait, certes d'une histoire acadienne marquée par le drame de la déportation et de la minorisation, mais surtout d'une participation active aux grands mouvements de révolte et de revendications qui marquaient les sociétés modernes ${ }^{2}$. Cette prise de parole des années 1970, ce moment d'affirmation et de modernisation du discours, a été si forte qu'elle a fini par se constituer en mythe dans le grand récit de l'Acadie. Depuis, aucune remise en question semblable ne s'est produite et les écrivains qui arrivent à l'écriture dans les années 1990 semblent avoir été condamné à occuper une place d' « éternel relève », c'est-à-dire d'une génération qui arrive après un moment fort sans pouvoir s'en démarquer. François Paré signalait déjà au milieu des années 1990 que les écrivains « des années 90 appartiennent d'emblée à une histoire littéraire, à laquelle ils répondent, dont ils se jouent, qu'ils rejettent, qu'ils réinterprètent à leur tour, dont ils révèlent les échecs et les fautes : cette institution, qu'elle soit acceptée ou rejetée par eux, reste un puissant intertexte » (PARÉ, 1994 : 16). Comment se différencier quand l'intertexte prend toujours l'avant-plan du texte lu? Benoit Doyon-Gosselin remarquait justement, en 2005, que les écrivains de la relève depuis la fin des années 1990 -, malgré une forte production, devaient encore trouver leur propre voix : «[...] il me semble que la plupart des auteurs sont rendus à la croisée des chemins. Pour mieux se positionner dans le champ littéraire, ils devront faire des choix

\footnotetext{
${ }^{2}$ Par exemple, la modernisation de la société québécoise au cours de la Révolution tranquille, les révoltes étudiantes de mai 1968, les mouvements de grève en France, les tensions raciales aux États-Unis, l'assassinat de Martin Luther King, les manifestations contre la guerre du Viêt-Nam, etc.
} 
esthétiques qui leur permettront de se démarquer. Sinon, les recueils se suivront comme les recensions. » (DOYON-GOSSELIN, 2005 : 40) Il s'agirait donc, pour la relève, de faire entendre une parole qui leur appartienne, mais faut-il encore que cette parole soit entendue.

Je propose, dans la suite de cet article, d'aborder le recueil Une lettre au bout du monde (2001) de Jean-Philippe Raîche en ramenant le sujet du texte en avant-plan, c'est-à-dire en le dégageant le plus possible de l'intertexte qu'impose d'emblée la participation du texte à une littérature culturellement marquée. Ce recueil se prête bien à ce type d'exercice puisque, malgré qu'il ait été remarqué par la critique à sa parution (il a été en lice pour le prix Antonine-Maillet, le prix Émile-Nelligan et le prix du Gouverneur général), il ne fait l'objet d'aucune étude littéraire. Comme si le texte, qui ne plonge pas systématiquement le lecteur dans une réalité collectivement connotée, celle d'une Acadie moderne face à une Acadie folklorique, ne parvenait pas à se faire entendre. Pourtant, l'acadianité du sujet n'est pas complètement absent du recueil de Raîche, mais elle est repensée à travers le désir du sujet de se découvrir ailleurs, à travers l'autre. L'acadianité devient un point de départ qui permet au sujet d'accéder à un monde où il peut exprimer sa différence certes en tant qu'Acadien, mais également en tant qu'individu qui, recentré sur lui-même, en quête de l'autre ou, plutôt, du soi dans l'autre, creuse au fond de lui-même pour trouver une sorte de complétude avec le monde - au sens large - qu'il habite. Le recueil se veut, peut-on lire sur la quatrième de couverture, « le carnet de voyage d'un sédentaire » qui vacille entre l'introspection du soi et la fuite vers l'autre - qui n'est jamais clairement identifié - qui ouvre le soi à une parole universelle :

Entre l'abîme et l'invention de l'autre, se superposent et se confondent des êtres, des lieux et des époques. S'ouvrant sur le constat d'un échec, cette suite revient sans cesse sur d'impossibles promesses, invoquant la lumière et l'espace, la rupture et le geste, 
JIMMY THIBEAULT, «Entre acadianité et mondialisation : l'expression du " soiAcadien " à la rencontre de l'autre dans Une lettre au bout du monde de Jean-Philippe Raîche »

pour faire de la parole l'ultime asile du corps et de ses certitudes (RAîcHE, 2001, quatrième de couverture).

Cette description pose bien le sujet au cœur des poèmes du recueil. Ce sont les préoccupations intimes qui habitent le poète, non des préoccupations collectives qui ramèneraient le sujet à son attachement identitaire. Peut-on lire cette description comme un repli narcissique du poète sur lui-même? Ou est-ce simplement la quête de sens du soi dans ce qu'il est au plus profond de lui-même, dans ses aspirations et dans ses échecs, hors du cadre traditionnel qui le définit d'emblée. S'il s'attache à l'autre, s'il rêve de l'ailleurs, c'est qu'il y trouve une voie d'accès à une nouvelle profondeur de son être, et surtout à une parole pour en rendre compte, comme le laisse entendre la citation de Claude Beausoleil en ouverture de la première partie du recueil : «Je suis un voyageur que le langage invente » (cité dans RAÎCHE, 2001, p. 7). Le « détachement» ne signifie pas ici un désengagement à l'égard de l'Acadie, mais bien un détachement qui permet au soi de se réinventer dans un au-delà de son identité première, dans une parole qui lui permettrait d'aller au fond de lui-même et de se dire dans la totalité du soi, dans son individualité et dans son acadianité.

C'est donc moins à la représentation d'une parole strictement acadienne que je m'intéresserai qu'à la construction du sujet dans l'expression de son individualité et de l'ouverture qu'il démontre pour une certaine intégration du soi à l'universel. J'examinerai ainsi le parcours que suit le poète dans sa quête de trouver une sorte de totalité du soi, c'està-dire une manière de dire le soi qui ne se limiterait plus à une perception locale de lui-même mais qui se ferait dans un mouvement d'ouverture à un ailleurs et à un autre comme complétude de soi. Je suivrai le mouvement que décrit le poète depuis son détachement d'une certaine vision folklorique de lui-même à la réinvention du soi dans son rapport à l'autre : 
pour se dire dans le monde, le poète doit d'abord s'affirmer dans son individualité, dans son désir d'aller au-delà des limites de son identité, de se réinventer dans l'ailleurs au contact de l'autre, d'abolir la distance avec cet autre au point de se dire dans la complétude du nous. Il s'agira, au final, de voir comment cette parole en apparence détacher du sujet acadien comme on a pu le reprocher à plusieurs auteurs de la relève - permet de repenser l'inscription de l'acadianité dans l'imaginaire d'une relève en apparence individualisé.

Si la quête du soi ne signifie pas une rupture complète avec le premier lieu de son identité, il apparaît néanmoins nécessaire pour le poète d'en saisir les limites. Celles-ci se définissent d'abord dans la langue et dans la perception du monde qu'elle impose au soi. En ce sens, l'utilisation du dialecte acadien dans les premières pages du recueil sert à dire la distance qui rend le contact avec l'autre difficile. Comment, en effet, entrer en communication avec quiconque ne partage pas « la même langue / ni les mêmes angoisses / ni la même façon / de regarder la mer » (RAîcHE, 2001, p. 10)? Comment parler de « tous les testaments / que vous ne savez lire / pour ces noms de famille / s'écrivant suivant la musique / que les curés n'écoutaient pas » (p. 11) si l'autre ne possède pas les clés pour décoder la langue du soi? Car il y a bien, chez le poète, la conscience d'une certaine incommunicabilité de son être qui repose sur une incompréhension de sa langue, ce qui a pour effet de l'enfermer dans un discours construit de manière à rester inaccessible pour l'autre. Ainsi, lorsqu'il affirme « je vous écris du bout du monde / avec ma langue qui résonne » et qu'il passe d'une langue plutôt normative à une langue qui se parle « au son des leaps de track / des j'ma rappelle / des viens ouère 'icitt' ta là » (p. 10), le sujet semble conscient de s'enfermer dans un lieu inaccessible pour l'autre, dans « une langue barbare / où le monde n'est rien / loin de l'enfance et de vos 
JIMMY THIBEAULT, «Entre acadianité et mondialisation : 1'expression du " soiAcadien " à la rencontre de l'autre dans Une lettre au bout du monde de Jean-Philippe Raîche »

chants » (p. 11). L'incommunicabilité est d'autant plus marquée que le poète avoue, à la fin de cette section de deux pages écrites en acadien, que le passage à l'écrit de sa langue reste incertain, même pour lui qui peut en déchiffrer les codes : «je ne sais pas non plus comment s'écrit beugé » (p. 11). Or, si l'orthographe du mot pose problème au poète, qu'en est-il pour l'autre qui ne partage pas la même langue? L'utilisation du mot dans les vers qui précèdent cet aveu empêche effectivement les non-initiés de bien saisir le sens du message que tente de passer le poète :

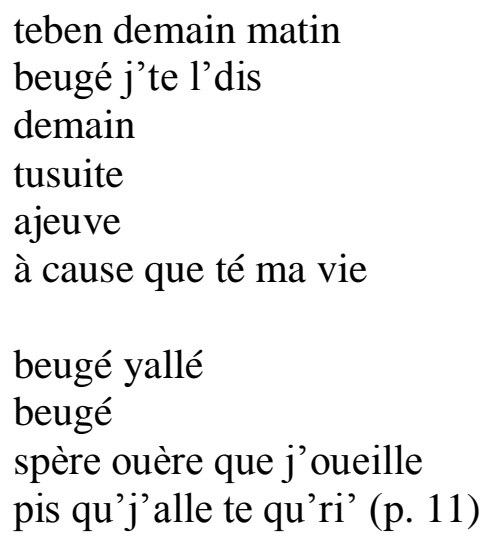

Cette langue hermétique que l'autre ne comprend pas et qui ne s'écrit pas vient en quelque sorte emmurer le poète dans un silence qui étouffe, lui qui est pourtant en quête de parole :

j'ai cherché dans les corps

le sublime et l'enfance

car je voulais toucher des mots

j’ai cru m’y élever

j'arpentais mes silences de sourd. (p. 15)

C'est d'ailleurs dans cette « langue barbare » qu'il affirme l'urgence qu'il ressent de fuir vers un ailleurs indéterminé, « l'ôte bôrd du clos / p'us loin que l'large / ayoù c'que rien nous bâd'e » (p.10). Pour le poète, sa langue, incomprise de l'autre, devient donc le lieu d' « une 
immense solitude / entre l'homme et le monde » (p. 60), le lieu d'une étrangeté qui empêche le soi de se dire à l'autre et de se dire dans l'autre.

Franchir les limites de la langue première permet ainsi au poète de briser le cadre de son identité pour rejoindre un espace autre où il lui sera possible de se réinventer. En fait, tout se passe comme si la description de soi, dans sa propre langue, n'offrait qu'une vision incomplète de lui-même parce que faite sur le mode de l'exclusion. Sortir de cette langue, aller vers l'ailleurs, vers l'autre, serait ainsi une manière d'avoir accès à une vision du monde plus large, une manière aussi d'intégrer ce monde en se le rendant présent dans sa totalité. Il s'agit, en quelque sorte, d'apprendre à nommer le monde habité différemment afin d'en saisir toute la profondeur. Tout en s'inscrivant dans une langue autre pour se dire, le sujet en vient à se réinventer en précisant le sens du soi. L'autre joue alors un rôle important dans ce processus qui amène le poète à se redéfinir puisqu'il devient, interlocuteur étranger, la raison du retour sur soi. Pour cet autre, le soi est un «paradigme absent»-pour prendre une expression de Marc Angenot (1978) : un mot dont le sens reste inconnu tant qu'il n'est pas défini, expliqué et présenté dans sa nature profonde. Le poète en arrive donc à se rendre compréhensible pour l'autre en faisant le récit de sa « violence secrète » (RAÎCHE, 2001, p. 9), de sa «honte»(p. 11) et de ses «naufrages» (p. 17). Autrement dit, en se racontant à l'autre, dans la langue de l'autre, dans un lieu autre, le poète apprend lui-même à se définir et à mieux saisir son identité intime, et ce, même dans les mensonges contés aux amants de passage. En un sens, le poète représente bien ce « voyageur que le langage invente » auquel la citation de Beausoleil fait référence en ouverture du recueil, de sorte que le voyage, prétexte à la reformulation du soi, apparait comme un voyage identitaire au sens où il est possible de le définir avec Éric Landowski : 
JIMMY THIBEAULT, «Entre acadianité et mondialisation : 1'expression du " soiAcadien " à la rencontre de l'autre dans Une lettre au bout du monde de Jean-Philippe

Raîche »

De ce point de vue, toute construction identitaire, toute « quête de soi », passe par un procès de localisation du monde - du monde comme altérité et comme présence (plus ou moins «présent») par rapport à soi. Et inversement, toute exploration du monde, tout «voyage », en tant qu'expérience du rapport à un ici-maintenant sans cesse à redéfinir, équivaut à un procès de construction du je (Landowski, 1997, p. 91)

Si la sédentarité du sujet, prisonnier de sa langue, limitait sa présence au monde, le voyage lui permet de briser la distance entre le soi et l'autre, entre l'ici et l'ailleurs, et de donner un nouveau sens à un monde qu'il habitait pourtant dans sa langue.

Le changement de paradigme qui se produit au fil des voyages et des rencontres repose sur un changement de perspective adopté par le sujet. Pour rompre avec la distance entre le soi et l'autre, pour faire de l'ailleurs un ici au soi, pour nommer le monde hors de sa langue, le sujet doit d'abord acquérir la langue de l'autre et saisir la perception qu'a ce dernier à la fois du monde et du soi. Le poète d'Une lettre au bout du monde en est bien conscient alors qu'il apprend à sentir le monde différent à travers le regard et le corps de l'autre. L'éveil des sens du poète, en donnant justement un nouveau sens à la place du soi dans le monde, devient le moteur de l'écriture qui n'avait été jusque-là qu'une vaine tentative sans véritable profondeur. Il avoue, à ce propos, n'avoir « pour ainsi dire rien écrit » avant la rencontre de l'autre, que

des pages entières raturées où s'alignaient les rythmes superbes, mais sans vie; des vers qu'on avait lus, y décelant parfois un talent dont on avait loué la jeunesse. Rien sinon cette phrase au souffle long, aux images crues, qui lui était venu un soir après que se soient estompés tous les noms qu'avait portés la nuit. Il se rappelait cette phrase sans effort comme s'il l'eut connue avant même d'en trouver les mots et se la répétait souvent pour en saisir le secret, mais en vain : son corps seul en avait senti la beauté. (RAÎCHE, 2001, p. 23)

Pour le poète, la faiblesse de son écriture repose en quelque sorte sur la faiblesse de son regard, de ses expériences qui ne lui permettent pas de trouver les mots pour dire ce que le 
corps ressent. C'est ainsi qu'il affirme vouloir écrire le monde par le regard des autres : «C'est ce regard que je voudrais écrire, pour me rappeler vos yeux, les yeux des autres, la forme mère de tant de lèvres qu'un nom parfois caractérise, qu'un geste fixe lorsqu'elles sont sur le point de s'offrir à la parole, parfaites de déséquilibre et d'inventions, amoureuses, tournées vers un corps impatient que la quête a tendu. » (p. 23) Ce qui lui manque cependant pour y parvenir, ce sont les mots qui donnent à la parole la force de s'inventer dans ce regard autre, car ses mots à lui ne suffisent pas :

A demi-mot, je me suis raconté vaguement, presque silencieusement, les yeux mi-clos comme en jouissant, de la hantise à la honte à la peine. Je n'ai rien dit. Ni de la rage, ni de la solitude. Il avait vingt ans de trop. J'ai voulu tuer le silence qui l'habitait dans tous mes rêves et j'ai parlé trop longuement.

J'aurais dû retrouver ma superbe et l'habitude paresseuse des mauvais poèmes, être lâche et lui dire bêtement que j'étais amoureux. (p. 25)

La véritable rupture n'est peut-être pas le départ du sujet, mais l'impossibilité pour lui de rejoindre l'autre, de « le poursuivre dans le couloir du train [... c] $]$ omme s'amorcerait un mouvement longtemps couvé par la douleur et qui pourtant n'aurait aucune identité, aucun sens, ne répondrait à aucune logique, ne confirmerait rien » (p. 25). Autrement dit, il faut se défaire des limites du soi pour atteindre un sens inaccessible dans sa langue : «J'ai appris tant de mots, mais jamais à parler, jamais à dire la beauté de ton ombre ou sur mes doigts le jour te cherchant à l'autre bout du monde. » (p.26)

Cette parole, ce dire, le poète l'acquiert au fil des voyages qui le conduisent dans « les terres du Sud », «plus loin que l'hiver. Au pied des grandes forteresses » et dans « les villes saintes » qui a vu « se dresser dans la splendeur des déserts au soleil »(p.26) : « J'ai entendu les mots étranges et beaux des langues d'ailleurs venues des empires magnanimes, des campagnes sans nom, du bout du monde. » (26) Ce n'est qu'une fois la route parcourue, les 
JIMMY THIBEAULT, «Entre acadianité et mondialisation : 1'expression du " soiAcadien " à la rencontre de l'autre dans Une lettre au bout du monde de Jean-Philippe

Raîche »

paysages et les mots étrangers intégrés à l'imaginaire intime, que le poète abolira finalement

la distance avec l'autre et qu'il lui sera possible de dire une parole qui se forme au-delà de la langue. S'il affirme effectivement «Tout ce que j'ai vu m'a rapproché de toi », c'est le contact des corps qui donnera véritablement sens à la place qu'occupe le soi dans l'ici et le maintenant :

À toi mes paumes moites et puis ma peau dont tu redis le goût quand tu gémis le jour, quand tu m'envoûtes et me donnes au midi froid d'hiver, quand tu m'amènes, une heure, nos rires de ventres chauds, pour m'apprendre à parler sur ton corps. (p.26)

L'union des corps permet au sujet de comprendre que c'est dans la rencontre de l'autre que le soi parvient à se dire dans sa totalité, dans sa complétude, et à intégrer un monde qui n’est «jamais fixe», mais qui prend forme «dans l'idée de la marée lorsqu'elle se tient en équilibre au bout des lèvres » (p.27). L'autre devient alors l'élément manquant dans la phrase que compose le poète pour marquer sa présence au monde. Avec la présence de l'autre, l'écriture est rendue possible pour le poète puisque la phrase fait désormais sens :

Tu restes un nom que j'écris à voix haute en descendant la rue comme en la remontant, le matin comme le soir depuis déjà l'enfance. Une voix, quelques images qui se ressemblent et dont j'ai pu déduire une attitude ou le phrasé d'un geste, ou peut-être un regard s'animant à l'approche de l'autre, de celui qu'on connaît pour l'avoir attendu tout un jour. Tu es surtout la moitié d'une phrase commencée dans ce qui me semblait alors de l'insouciance comme un appel à jouer, comme un possible encore secret ne présumant de rien, avançant tel un enfant timide, osant peu, tel un risque rompu aux exigences inavouables de l'orgueil, aux artifices des retraites. Tu es la moitié d'une phrase que nous ferons mourir, ce compagnon de solitude heureuse, ce complice fébrile né de l'attente et du secret, de l'aveu et de l'impatience. Tu viendras et nos corps assoupis seront alors le seul poème. (p.27)

À travers l'autre, donc, dans la sexualité qui les unit, le soi se dévoile à lui-même et marque, par le fait même, sa présence au monde. 
Si la rencontre de l'autre permet au soi d'appréhender différemment la place qu'il occupe dans le monde, elle lui permet également de modifier la perception qu'il a du lieu de l'origine, qu'on devine être l'Acadie bien qu'elle ne soit jamais directement nommée. Le retour ne peut avoir lieu que sous le signe d'un changement perceptible uniquement dans le regard de celui qui a été absent pendant un certain temps et dont la vision a été suffisamment affectée pour saisir les subtiles variations de l'espace. Il est d'ailleurs significatif que la troisième partie du recueil soit mise sous l'égide d'une citation d'Herménégilde Chiasson qui fait état, justement, du temps qui passe et du monde qui change dans son sillage : « Les arbres sont plus longs qu'avant / Le plafond est plus blanc / Et la saison avance » (cité dans RAîcHE, 2001, p.29) Comme pour faire écho aux paroles de Chiasson, c'est par un regard étranger, «l'œil étonné / par la blancheur de janvier / qu'il n'avait jamais vue » (RAîcHE, 2001, p.31), qu'est décrit l'espace en ouverture de cette partie. Le poète joue ici avec l'ambiguïté du « il » qui peut certes renvoyer à l'amant étranger en visite pour la première fois dans les lieux de l'enfance du poète, mais il pourrait bien être aussi le poète lui-même regardant «janvier / dans le regard / d'un homme neuf » (p.31), donc avec le regard de celui qui a voyagé et qui n'est plus tout à fait d'ici :

Avant de s'endormir

il se couvrait de son manteau

pour ne pas oublier

qu'il était de passage

c'était une habitude

que lui avaient donnée les voyages

leurs lentes solitudes. (p.32)

Cette ambiguïté du sujet s'estompe ensuite alors que la troisième personne cède la place, après une page blanche, à l'acte de communication que le « je » établit avec le «tu », allant plus loin dans l'intimité des corps. 
JIMMY THIBEAULT, «Entre acadianité et mondialisation : 1'expression du " soiAcadien " à la rencontre de l'autre dans Une lettre au bout du monde de Jean-Philippe Raîche »

Le lieu joue-t-il un rôle dans cette intimité du couple? Sans doute, mais pas de la même manière que dans la poésie des poètes acadiens des années 1970, dans laquelle l'espace acadien devient un leitmotiv au récit du soi comme partie d'un «nous ». Dans le recueil de Raîche, il semble à première vue que l'espace ne compte pas réellement puisque, malgré le lieu, c'est vers l'autre que se tourne le regard du poète, jamais vers l'espace acadien. Tout se passe comme si le lieu n'avait d'importance que dans la présence de l'autre : «pourvu que l'aube passe / dans ton regard / sur moi. » (p.38). Pourtant, la relation que le poète entretient avec l'autre a bien un lien avec l'espace habité alors qu'ils vivent leur amour au rythme du Mascaret :
le Mascaret montant à nos moissons d'argile la Mascaret montant sur nos bouches tièdes sur nos envies d'hier nos promesses d'exil le Mascaret montant entre gelées de ciel le Mascaret monté de la terre à la mort de la mort à la mer monte le Mascaret monte le Mascaret (p.43-44)

L'espace, qu'il soit d'ici ou d'ailleurs, se décline donc selon les rapports que le soi entretient avec l'autre, il trouve dans la relation du couple un sens qui le définit au gré des souvenirs que le sujet interpelle. De sorte que le lieu, qui se remplit d'un nouveau sens dans le regard de celui qui le découvre pour la première fois, prend l'apparence des pages blanches qui marquent la troisième partie - aux pages 33,34, 45 et 46 -, donc vidée de son texte, lorsque le soi se retrouve seul après la rupture du couple. En ce sens, l'espace est comme la page ou 
comme l'écriture sur la page que le poète affirmait vide de sens jusqu'à ce qu'il rencontre l'autre. Ce n'est donc pas la mémoire du lieu qui nourrit l'écriture, mais la manière de l'habiter au présent :

comme les derniers échos

d'une incantation blanche

je reconnais les murmures du vieux peuple

je me souviens que je meurs à l'autre bout du monde

dans le corps des dieux

et qu'ici

je suis seul. (p.54)

L'absence de l'autre, qui plonge le poète dans la solitude du quotidien, influence la représentation des lieux alors que, dans le village, les

héritages bricolés

de bois mort ou vivant

avec leurs lettres peintes

qui ont nommé le quotidien

qui s'usent

et qui s'effaceront (p. 47)

L'écriture, mémoire du village, s'effacera de la même manière que s'effaceront les souvenirs que garde de l'autre le poète, le goût de sa peau, son nom, sa présence et, ultimement, le sens qu'il donnait au soi; car le poète à fait de cet autre son «origine », sa « seule dette », son « refuge », « [s]a lâcheté / [s]on corps / [s]on voyage / et ses lignes de fuites » (p.56). Ainsi, lorsque le poète affirme «tu me survis / chaque jour / plus longtemps » (p.56), c'est qu'il comprend que le sens du soi s'efface toujours un peu plus dans la distance alors que les lettres qu'a tracé le soi au contact de l'autre s'estompent lentement pour ne laisser qu'une page blanche, vide, une phrase au sens incomplet : «Je vous appellerai de loin pour ne pas. » (p.62) 
JIMMY THIBEAULT, «Entre acadianité et mondialisation : 1'expression du " soiAcadien " à la rencontre de l'autre dans Une lettre au bout du monde de Jean-Philippe Raîche »

Dans les trois premières parties du recueil, les vers du poète n'auront toujours eu comme sujet que la quête du soi pour une parole lui permettant de se dire. L'autre apparaît alors comme une sorte de passeur qui donne au soi un accès à cette parole et au sens qui s'y cache. Ainsi, si c'est dans l'absence de l'autre que le langage du corps devient verbe et que l'écriture devient lieu de création poétique, il semble que la distance, comme je l'ai souligné plus tôt, ne peut que résulter en un effacement du sens que le poète avait découvert au fond de luimême. Le poète en est bien conscient et la quatrième partie repose sur une sorte d'inversion du rapport entre le soi et l'autre alors que le poète écrit son désir de rejoindre l'autre, pas uniquement pour trouver une complétude au soi, mais aussi pour compléter l'autre dans l'invention d'un « nous » relativement absent dans les premières parties du recueil. Il est significatif, en ce sens, que cette dernière partie soit mise sous l'égide d'une citation de Gérald Leblanc qui pose, justement, le verbe « aimer » au cœur de la relation entre les deux amants : «Ma bouche et mes mains sont pleines de toi / aimer est un verbe / et $\mathrm{j}$ 'en tremble » (cité dans RAîcHE, 2001, p.63). Il est significatif aussi qu'on retrouve dans les premières pages de cette partie une abondance de « nous » qui décrit, qui invente, l'union véritable du « je » avec le « tu ». Le poète affirme ainsi :

et si nous regardions la mer une première fois plus fous plus beaux plus vrais qu'avant (RAîCHE, 2001, p.67)

plus infinis que tous les fleuves nous donnerons aux mots la fragilité d'un corps un pays inventé par nos gorges sauvages nos gorges indomptées 
nous parlerons parlerons parlerons

des nuits qui tombent

au bout du monde

alors que nous naissons ensemble

corps contre corps

solitaires et nus (p.69)

Il n'est plus question, dans l'utilisation du « nous », d'un soi qui apprend à percevoir le monde à travers les sens de l'autre, mais plutôt d'un soi qui partage une expérience avec le «tu » afin d'en faire un moment de parfaite union. Dans les premières parties du recueil, il semble que le « soi » naît à lui-même, dans son corps. Au fil de la dernière partie c'est plutôt le « nous » qui naît dans un rapport de réciprocité entre les corps. Qu'importe le lieu finalement puisqu'il n'a de sens que par la double présence du soi et de l'autre qui rendent pluriel les mots « seuls » et « solitaires »? «Seul» ensemble, «solitaire » à deux, le langage que le poète aura appris au contact de l'autre sert finalement à abolir la distance entre les lieux de la mémoire et de l'imaginaire : « je sais depuis / que l'horizon est une longue phrase aux rythmes déployés / j’y tracerai tous nos itinéraires. » (p.71)

C'est dans ce désir d'abolir la distance qui le sépare de l'autre que le poète utilise donc le langage appris pour maintenir, du moins dans la mémoire, la présence rassurante de l'autre. Alors que le soi s'invente au contact de l'autre, c'est maintenant à son tour d'inventer l'autre par l'amour qui les unit et qui les complète. S'il avait peur, dans la troisième partie, d'oublier le nom de son amant, il peut désormais affirmer : « et si tu meurs / je te donnerai mon nom pour te réinventer. » (p.70) Car le poète est désormais prêt à donner à son tour à cet autre un sens qu'il invente et qu'il intériorise avec les mots appris : «je te répéterai ton nom // je goûterai sur ta peau neuve / le souffle tiède de mes mots / les nuages. » (p.78) Ce n'est, au final, que dans cette réciprocité des corps et du langage, dans les lieux de la mémoire et dans 
JIMMY THIBEAULT, «Entre acadianité et mondialisation : 1'expression du " soiAcadien " à la rencontre de l'autre dans Une lettre au bout du monde de Jean-Philippe Raîche »

la présence de l'imaginaire que le soi peut trouver le verbe pour construire sa parole, pour se dire, mais aussi pour dire le monde à travers l'autre qui vient compléter le «nous » du couple :

Je veillerai la nuit et les choses banales que le silence en aie les gestes

ceux que mon souffle attend ceux que je t'offrirai

pour qu'au matin tu fasses naître un vers à traverser comme une rue. (p. 80)

Le poète aura finalement compris le besoin de l'autre pour se raconter et, surtout, pour s'inventer, donnant ainsi du sens aux deux vers qui apparaissent en retrait de la deuxième partie : «J'ai laissé sur le seuil une lettre fermée / l'histoire que nous n'avons pas faite. » (p. 21) Ce qui rompt réellement la distance, c'est donc le sentiment d'avoir quelque part un interlocuteur à même de comprendre l'imaginaire se formant dans la parole intériorisée d'un «nous ».

J'ai formulé ailleurs l'hypothèse que les poètes de la longue décennie 1970 ont cherché à dire l'espace qu'ils habitent à travers les expériences individuelles qu'ils y vivaient : «En Acadie, [...] il n'est plus question de l'Acadie perdue, de l'espace mythique d'avant la déportation, mais d'une Acadie intime qui se définit dans le regard du poète; l'Acadie n'est plus ce qui définit le sujet, c'est désormais l'expérience et le regard du sujet qui définissent l'espace habité. » (ThIBEAULT, 2014-2015, p. 32) Les générations qui ont suivi ont intériorisé ce discours, ils ont intériorisé cette manière de percevoir leur acadianité, au point où leur regard 
s'est doublement porté au-delà de l'Acadie, au-delà de leur identité acadienne, en cherchant au-dedans d'eux-mêmes les particularités qui en font des individus occupant une place définie dans la société qu'ils intègrent; au-delà de la seule Acadie comme espace habité, en cherchant dans l'ailleurs et dans l'autre une parcelle de soi. Mais il ne faut pas voir ici le refus d'une parole identitaire qui a pris forme bien avant eux : au contraire on retrouve dans les recueils de la relève des traces qui les posent constamment dans le sillage de leurs devanciers, que ce soit par des références directes en exergue des œuvres, par l'intégration de la figure des grands poètes des années 1970 dans le corps des poèmes ou par les renvois intertextuels à leurs œuvres. Une lettre au bout du monde ne fait pas exception à cette règle puisque le poète revient toujours vers l'Acadie, vers le mascaret, que ce soit dans l'énonciation de son enfance ou dans le regard de l'autre qui donne à l'espace une couleur nouvelle. Il revient aussi vers la parole acadienne qui fait entendre son écho dans les citations d'Herménégilde Chiasson et de Gérald Leblanc placées en ouverture des troisième et quatrième parties du recueil. Certes, ces citations ne renvoient pas aux grands textes identitaires de ces auteurs, mais elles n'en portent pas moins la référence. En ce sens, le recueil de Jean-Philippe Raîche permet de repenser l'acadianité du sujet en faisant de cette référence identitaire une partie indéniable de son identité, mais pas son unique fondement. Au-delà des référents acadiens que le sujet a intériorisés dès l'enfance, il y a les expériences qu'il vit dans l'espace, l'ici et l'ailleurs, et dans la rencontre de l'autre, ces expériences lui permettent de découvrir des lieux inexplorés du soi et d'apprendre à s'appréhender dans sa totalité, hors des lieux communs qui réduisent le soi à une identité fixée dans son appartenance à un collectif. Le « nous », ici, n'est effectivement pas réductible à l'intégration 
JIMMY THIBEAULT, «Entre acadianité et mondialisation : 1'expression du " soiAcadien " à la rencontre de l'autre dans Une lettre au bout du monde de Jean-Philippe Raîche »

du soi à la collectivité, mais se construit sur la rencontre et, surtout, la communication d'un $\ll \mathrm{je} »$ avec un $\ll$ tu $»$.

En 1996, François Paré écrivait, au sujet de la production littéraire franco-ontarienne, mais l'affirmation valait également pour l'Acadie : « La question de l'identitaire a-t-elle fait son temps? La réponse est certes non, dans la mesure où elle n'est pas résolue - pas pour moi du moins - et où elle continue de hanter la conscience collective. Mais, du même souffle, il convient d'aller plus loin. » (PARÉ, 1996, p. 18) Plus de vingt ans plus tard, il me semble que la question demeure... de même que la réponse. Sinon, comment expliquer l'absence d'analyses de fond d'œuvres comme celle de Jean-Philippe Raîche? Pensons notamment à la poésie de Fredric Gary Comeau, Éric Cormier, Sarah Marylou Brideau ou, chez la génération plus récente, Sébastien Bérubé, Jonathan Roy et Monica Bolduc. Il semble que, lorsque la critique universitaire écrit sur eux, c'est toujours pour se demander s'ils forment une relève digne de ce nom à la poésie acadienne. Pour que la relève attire davantage l'attention, il faudra peut-être revenir de manière plus incisive sur la tension qui existe entre l'identité de la collectivité et celle du sujet acadien qui, à l'instar du sujet poétique d'Une lettre au bout $d u$ monde, cherche à faire entendre sa voix dans le maelström des voix planétaires, à communiquer avec elles et à s'affirmer en elles. Il faudra, je pense, trouver une manière de dire que, malgré cette tension dont on ne sait trop quoi faire, l'un n'empêche pas forcément l'autre et que ces voix qui s'élèvent cherchent plutôt à s'approprier leur acadianité et à l'élever au rang des voix universelles. En cela, le recueil de Raîche est exemplaire, mais fautil que la critique s'y attarde pour bien mettre en lumière cette manière de dire l'acadianité. Mais, finalement, le drame pour les auteurs qui écrivent à partir de la fin des années 1990 est peut-être que la génération de 1970 s'est instituée en figure mythique dont l'Acadie n'est pas 
prête à se départir comme elle l'a fait, du moins symboliquement, avec un certain folklore qui semblait emprisonner l'Acadie dans ses clichés. Le drame est peut-être aussi que la relève n'est elle-même pas prête à rompre avec ce «cri de terre » rauque qui résonne dans une douleur si belle qu'on voudrait la faire sienne.

\section{Bibliographie}

ANGENOT, Marc (1978), «Le paradigme absent : Éléments d'une sémiotique de la sciencefiction », Poétique, nº33, février, p. 74-79.

Delic, Emir et Jimmy ThiBEAUlt (2014-2015), «La poésie franco-canadienne de la longue décennie 1970 (1968-1985). Introduction », Francophonies d'Amérique, n³8-39, automneprintemps, p. 11-23.

DoYON-Gosselin, Benoit (2005), «Y a-t-il une relève en poésie acadienne? "Les nouveaux poètes acadiens à la croisée des chemins" », Liaison, $\mathrm{n}^{\circ}$ 129, p. 38-40.

Landowski, Éric (1997), Présences de l'autre. Essais de socio-sémiotique II, Paris, Presses universitaires de France.

PARÉ, François (1994), Théories de la fragilité, Ottawa, Le Nordir.

PARÉ, François (1996), «Pour rompre le discours fondateur : la littérature et la détresse », dans Lucie Hotte et François Ouellet (dir.), La littérature franco-ontarienne: Enjeux esthétiques, Ottawa, Le Nordir, p. 11-26.

RAÎCHE, Jean-Philippe (2001), Une lettre au bout du monde, Moncton, Perce-Neige.

ThiBEAUlt, Jimmy (2014-2015), « La prise de parole poétique de la longue décennie 1970 : une trace de la franco-américanité », Francophonies d'Amérique, no 38-39, automneprintemps, p. 25-47.

\section{Résumé}

Cet article propose une étude du rapport qu'entretient le poète du recueil Une lettre au bout de monde de Jean-Philippe Raîche avec la figure de l'autre incarné par l'amant étranger. Ce rapport se construit dans le désir de rapprochement et de mise en communication qui oblige 
JIMMY THIBEAULT, «Entre acadianité et mondialisation : 1'expression du " soiAcadien " à la rencontre de l'autre dans Une lettre au bout du monde de Jean-Philippe

Raîche »

le soi à se repenser dans le monde. Entre l'acadianité du sujet et son désir de rejoindre le monde, le poète raconte le parcours qui l'amène à se redéfinir dans son intimité : dégagé dès le début du recueil des a priori qui le fixent dans son identité acadienne, le poète part à la rencontre de l'autre dans une quête qui s'avère finalement celle du soi.

\begin{abstract}
Abstact
This article proposes a study of the relationship between the poet in Jean-Philippe Raîche's Une lettre au bout de monde with the figure of the other embodied by the foreign lover. This relationship is built in the desire for rapprochement and communication that forces the self to rethink his place in the world. Between the subject's "acadianité" and his desire to join the world, the poet tells the story that leads him to redefine himself in his intimacy: released from the beginning of the book of a priori that fixes it in his Acadian identity, the poet leaves to meet the other in a quest that ultimately turns out to be the self.
\end{abstract}

\title{
Notice biobibliographique
}

Jimmy Thibeault est professeur agrégé au Département des études françaises de l'Université Sainte-Anne où il est titulaire, depuis 2013, de la Chaire de recherche du Canada en études acadiennes et francophones. Il enseigne les littératures acadienne, québécoise, francoontarienne et francophone de l'Ouest. Ses travaux portent sur la représentation des enjeux identitaires, individuels et collectifs, dans les espaces culturels francophones du Canada. Il s'intéresse également aux transferts culturels en contexte de migration, de continentalité et de mondialisation. En 2015, il a fait paraître Des identités mouvantes : Se définir dans le contexte de la mondialisation (Éditions Nota bene, Prix Gabrielle-Roy 2015), un ouvrage qui aborde ces problématiques. Il a aussi publié de nombreux articles savants et chapitres d'ouvrages collectifs sur les littératures francophones du Canada. Il a codirigé des dossiers spéciaux de revue dans Voix et Images (2011), @ nalyses (2011), Québec Studies (2012) et Francophonies d'Amérique (2014-2015), ainsi que l'ouvrage collectif Au-delà de l'exiguïté. Échos et convergences dans les littératures minoritaires (Éditions Perce-Neige, 2016). 